\title{
Finite-time robust stabilization of uncertain delayed neural networks with discontinuous activations via delayed feedback control
}

\author{
Leimin Wang ${ }^{\mathrm{a}, \mathrm{b}}$, Yi Shen ${ }^{\mathrm{a}, \mathrm{b}, *}$, Yin Sheng ${ }^{\mathrm{a}, \mathrm{b}}$ \\ ${ }^{a}$ School of Automation, Huazhong University of Science and Technology, Wuhan 430074, China \\ ${ }^{b}$ Key Laboratory of Image Processing and Intelligent Control of Education Ministry of China, Wuhan 430074, China
}

\begin{abstract}
This paper is concerned with the finite-time robust stabilization of delayed neural networks (DNNs) in the presence of discontinuous activations and parameter uncertainties. By using the nonsmooth analysis and control theory, a delayed controller is designed to realize the finite-time robust stabilization of DNNs with discontinuous activations and parameter uncertainties, and the upper bound of the settling time functional for stabilization is estimated. Finally, two examples are provided to demonstrate the effectiveness of the theoretical results.
\end{abstract}

Keywords: Delayed neural networks (DNNs), finite-time robust stabilization, discontinuous activations, parameter uncertainties, delayed controller, settling time.

\section{Introduction}

In recent years, stability of neural networks (NNs) has received great attention for its potential applications in pattern recognition, parallel computation, associative memory and control optimization (Cao \& Wang, 2005; Cohen \& Grossberg, 1983; Forti \& Tesi, 1995; Shen \& Wang, 2009; Zeng \& Zheng, 2012). As is well known, during the hardware implementation of NNs, time delays are unavoidable due to the finite switching speed of the neuron amplifiers and they often cause undesirable dynamical behaviors such as instability or oscillation. On the other hand, the parameters of NNs may exhibit some deviations because of the existence of modeling errors, external disturbance, and parameter fluctuations, which would cause the parameter uncertainties. Therefore, it is of practical interest to take into account the time delays and parameter uncertainties when studying stability of NNs. In other words, the robust stability problem of delayed neural networks (DNNs) should be considered.

Recently, robust stability of DNNs has been widely investigated and various robust stability criteria have been proposed (Arik, 2014; Faydasicok \& Arik, 2012; Feng et al., 2015; Guo \& Wang, 2014; Guo \& Huang, 2009; Huang et al., 2012; Shen \& Wang, 2012; Wang \& Li, 2014; Wu et al., 2010; Xiao \& Zeng, 2014; Yang et al., 2009; Zhang et al., 2008; Zuo et al., 2009). However, the neuron activations considered in Arik (2014); Faydasicok \& Arik (2012); Feng et al. (2015); Guo \& Wang (2014); Huang et al. (2012); Shen \& Wang (2012); Wang $\&$ Li (2014); Yang et al. (2009); Zhang et al. (2008) are continuous, or even Lipschitzian. The authors in Forti \& Nistri (2003);

\footnotetext{
${ }^{*}$ Corresponding author. Tel.: +86 27 87543630; fax: +86 2787543130 Email addresses: leiminw89@126.com (Leimin Wang), yishen64@163.com (Yi Shen)
}

Preprint submitted to Neural Networks
Forti et al. (2005) have demonstrated the interest in studying the stability problem of DNNs with discontinuous activation functions. The discontinuous activations are in the case that the gain of the neuron amplifiers is very high and the analysis of the ideal discontinuous case can better reflect the crucial features of the dynamics. It is shown that DNNs with discontinuous activations are frequently encountered in practice, and the analysis of the discontinuous case deserves the research interest in the potential applications of dry friction, switching in electronic circuits, optimization problems (Forti \& Nistri, 2003; Forti et al., $2005,2006)$. Thus, it is necessary to consider the discontinuous activations for more general applications of DNNs.

Different from the asymptotical stabilization with infinite settling time, finite-time stabilization gives the convergence with finite settling time. It requires essentially that a control system is Lyapunov stable and its trajectories tend to zero in finite time under the designed controller. Previously, Bhat \& Bernstein (2000) proved that there is a necessary and sufficient condition for finite-time stability of multi-dimensional continuous autonomous system. Since then, the problems of finitetime stability and stabilization have been widely studied (Efimov et al., 2014; Forti et al., 2005; Hong \& Jiang, 2006; Hu et al., 2014; Huang \& Li, 2014; Karafyllis, 2006; Liu et al., 2014a,b; Moulay et al., 2008; Sun et al., 2014; Wang \& Xiao, 2010; Wang et al., 2015b; Wang \& Shen, 2015; Yang, 2014). However, so far few studies have been published concerning finite-time stability of time-delay systems (Moulay et al., 2008; Yang \& Wang, 2012). This is because time-delay systems exhibit more complicated dynamic behaviors and they are more difficult to deal with than system without delays. As stated in Moulay et al. (2008), it is difficult to find a Lyapunov functional that satisfies the derivative condition for the finite-time stability of time-delay systems. Thus, the finite-time stability of time-delay systems, especially for the DNNs, is still an open 
problem that requires further investigation.

Motivated by the above discussions, we study the finite-time stability problem of NNs by taking into consideration the time delays, parameter uncertainties and discontinuous activations in our paper. To the best of authors' knowledge, so far there is few results for the finite-time stabilization of DNNs with discontinuous activations and parameter uncertainties. By using a designed delayed feedback controller, the finite-time robust stabilization for this system is realized. The contributions of this paper are as follows.

1) Time delays, discontinuous activations and parameter uncertainties are considered in studying the finite-time stability problem.

2) A delayed feedback controller is designed to realize the finite-time robust stabilization for a class of DNNs with discontinuous activations and parameter uncertainties.

3) The NNs model in our paper is in presence of parameter uncertainties and discontinuous activations, which makes our robust results more general than (robust) stability of neural networks with continuous activations in Arik (2014); Faydasicok \& Arik (2012); Feng et al. (2015); Guo \& Wang (2014); Shen \& Wang (2012); Wang \& Li (2014); Cao \& Wang (2005); Liu \& Wang (2006); Wang \& Shen (2014).

4) The finite-time stabilization with finite settling time improve and extend the stabilization with infinite settling time in the previous works (Huang et al., 2013; Phat \& Trinh, 2010; Wang \& Li, 2014; Wen et al., 2015; Wu \& Zeng, 2012; Zhang $\&$ Shen, 2015).

5) In Liu et al. (2014a,b), finite-time stabilization was studied while the systems are without delays and parameter uncertainties. So our methods are general and can be used to study finite-time stability of other delayed systems with or without parameter uncertainties.

The remainder of this paper is organized as follows. Some preliminaries are introduced in Section 2. In Section 3, we design a delayed controller with which the finite-time robust stabilization of DNNs with discontinuous activations and parameter uncertainties is achieved. Besides, we estimate the upper bound of the settling time functional and provide the finite-time stabilization algebraic criteria for DNNs without parameter uncertainties. Then, two examples are provided to demonstrate the effectiveness and superiority of the obtained results in Section 4. Finally, conclusions are drawn in Section 5.

\section{System description and preliminaries}

The following notations will be used throughout this paper. $\mathcal{R}_{+}, \mathcal{R}^{n}$ and $\mathcal{R}^{n \times n}$ denote the set of all nonnegative real numbers, the $n$-dimensional Euclidean space and the set of all $n \times n$ real matrices, respetively. For all $x=\left(x_{1}, \ldots, x_{n}\right)^{T} \in$ $\mathcal{R}^{n},\|x\|=\sqrt{x^{T} x}$ is the Euclidean norm and $\operatorname{sgn}(x)=$ $\left(\operatorname{sgn}\left(x_{1}\right), \ldots, \operatorname{sgn}\left(x_{n}\right)\right)^{T}$ is the sign function. For a given square matrix $A=\left(a_{i j}\right)_{n \times n} \in \mathcal{R}^{n \times n},|A|=\left(\left|a_{i j}\right|\right)_{n \times n} . C\left([a, b], \mathcal{R}^{n}\right)$ denotes the space of all continuous functions $\Psi:[a, b] \rightarrow \mathcal{R}^{n}$ with uniform norm $\|\Psi\|=\sup _{a \leq s \leq b}\|\phi(s)\|$. A continuous function $v: \mathcal{R} \rightarrow \mathcal{R}$ belongs to the class $\mathcal{K}$ if it is strictly increasing and $v(0)=0$.
In this paper, we consider a class of DNNs as follows:

$$
\dot{x}(t)=-D x(t)+A f(x(t))+B g(x(t-\tau(t))),
$$

where $x(t)=\left(x_{1}(t), x_{2}(t), \ldots, x_{n}(t)\right)^{T} \in \mathcal{R}^{n}$ is the state vector. $D=\operatorname{diag}\left(d_{1}, d_{2}, \ldots, d_{n}\right)$ is a diagonal matrix with $d_{i}>$ $0(i=1,2, \ldots, n) . A=\left(a_{i j}\right)_{n \times n}, B=\left(b_{i j}\right)_{n \times n} \in \mathcal{R}^{n \times n}$ are the connection weight matrix and delayed connection weight matrix, respectively. $f(x(t))=\left(f_{1}\left(x_{1}(t)\right), \ldots, f_{n}\left(x_{n}(t)\right)\right)^{T} \in \mathcal{R}^{n}$ and $g(x(t-\tau(t)))=\left(g_{1}\left(x_{1}\left(t-\tau_{1}(t)\right)\right), \ldots, g_{n}\left(x_{n}\left(t-\tau_{n}(t)\right)\right)\right)^{T} \in \mathcal{R}^{n}$ are the neuron activation functions. $\tau(t)=\left(\tau_{1}(t), \tau_{2}(t), \ldots, \tau_{n}(t)\right)^{T}$ is the time-varying delay, which satisfies $0 \leq \tau_{j}(t) \leq \tau, j=$ $1,2, \ldots, n$.

An important factor that affects the stability is the uncertainties of network parameters. In this paper, the parameters matrices $D=\operatorname{diag}\left(d_{1}, d_{2}, \ldots, d_{n}\right), A=\left(a_{i j}\right)_{n \times n}, B=\left(b_{i j}\right)_{n \times n}$ of system (1) are assumed to be norm-bounded within the following ranges:

$$
\begin{array}{r}
D_{I}=[\underline{D}, \bar{D}]=\left\{D=\operatorname{diag}\left(d_{i}\right): 0<\underline{d}_{i} \leq d_{i} \leq \bar{d}_{i}, i=1,2, \ldots, n\right\}, \\
A_{I}=[\underline{A}, \bar{A}]=\left\{A=\left(a_{i j}\right)_{n \times n}: \underline{a}_{i j} \leq a_{i j} \leq \bar{a}_{i j}, i, j=1,2, \ldots, n\right\}, \\
B_{I}=[\underline{B}, \bar{B}]=\left\{B=\left(b_{i j}\right)_{n \times n}: \underline{b}_{i j} \leq b_{i j} \leq \bar{b}_{i j}, i, j=1,2, \ldots, n\right\} .
\end{array}
$$

The following assumptions are given for system (1).

(A1) For every $j=1,2, \ldots, n, f_{j}, g_{j}: \mathcal{R} \rightarrow \mathcal{R}$ are continuous except on a countable set of isolate points $\left\{\rho_{k}^{j}\right\}$, where the finite right and left limits $f_{j}^{+}\left(\rho_{k}^{j}\right), g_{j}^{+}\left(\rho_{k}^{j}\right)$ and $f_{j}^{-}\left(\rho_{k}^{j}\right), g_{j}^{-}\left(\rho_{k}^{j}\right)$ exist, respectively.

(A2) For each $j=1,2, \ldots, n$, suppose $0 \in K\left[f_{j}(0)\right], 0 \in$ $K\left[g_{j}(0)\right]$ and there exist constants $h_{j}>0, l_{j}>0, r_{j}>0, s_{j}>0$, such that

$$
\sup \left|\xi_{j}\right| \leq h_{j}|u|+r_{j}, \quad \sup \left|\zeta_{j}\right| \leq l_{j}|v|+s_{j},
$$

for all $u, v \in R$, where $\xi_{j} \in K\left[f_{j}(u)\right]=$ $\left[\min \left\{f_{j}^{-}(u), f_{j}^{+}(u)\right\}, \max \left\{f_{j}^{-}(u), f_{j}^{+}(u)\right\}\right], \zeta_{j} \in K\left[g_{j}(v)\right]=$ $\left[\min \left\{g_{j}^{-}(v), g_{j}^{+}(v)\right\}, \max \left\{g_{j}^{-}(v), g_{j}^{+}(v)\right\}\right]$.

Because of the presence of discontinuous activations, system (1) is discontinuous and its classical solution does not exist. Now we introduce the concept of Filippov solution (Filippov, 1988).

Definition 1 (Filippov, 1988). For a system with discontinuous right-hand side:

$$
\dot{x}(t)=F\left(t, x_{t}\right), t \geq 0,
$$

where $x(t) \in \mathcal{R}^{n}, x_{t} \in C\left([-\tau, 0], \mathcal{R}^{n}\right)$ and $x_{t}(s)=x(t+s),-\tau \leq$ $s \leq 0 . F:[0,+\infty) \times \mathcal{R}^{n} \rightarrow \mathcal{R}^{n}$ is Lebesgue measurable and locally essentially bounded. An absolutely continuous function $x(t), t \in[0, T], T>0$ is said to be a Filippov solution of system (4) with initial condition $x(s),-\tau \leq s \leq 0$, if it satisfies the differential inclusion:

$$
\dot{x}(t) \in \Phi\left(t, x_{t}\right), \text { for a.a. } t \in[0, T],
$$

where the Filippov set-valued map $\Phi\left(t, x_{t}\right):[0,+\infty) \times \mathcal{R}^{n} \rightarrow 2^{\mathcal{R}^{n}}$ is defined by

$$
\Phi\left(t, x_{t}\right) \triangleq \bigcap_{\delta>0} \bigcap_{\mu(N)=0} K\left[F\left(t, B\left(x_{t}, \delta\right) \backslash N\right)\right],
$$


$K[E]$ is the closure of the convex hull of set $E, E \subset \mathcal{R}^{n}$, $B\left(x_{t}, \delta\right)=\left\{y_{t}:\left\|y_{t}-x_{t}\right\|<\delta, x_{t}, y_{t} \in \mathcal{R}^{n}, \delta \in \mathcal{R}_{+}\right\}$, and $N \subset \mathcal{R}^{n}$, $\mu(N)$ is the Lebesgue measure of set $N$.

Note that since $F\left(t, x_{t}\right)$ is Lebesgue measurable and locally essentially bounded, $\Phi\left(t, x_{t}\right)$ is upper semicontinuous and has nonempty, compact, and convex values (Filippov, 1988). Thus, there exists a Filippov solution of system (4) (Filippov, 1988).

Next we extend the concept of the Filippov solution to system (1) as follows.

Definition 2 (Forti et al., 2005). A function $x:[-\tau, T) \rightarrow$ $\mathcal{R}^{n}, T \in(0,+\infty]$, is a solution (in Filippov's sense) of the discontinuous system (1) on $[-\tau, T)$, if:

i) $x$ is continuous on $[-\tau, T)$ and absolutely continuous on $[0, T)$;

ii) there exist two measurable functions $\alpha=$ $\left(\alpha_{1}, \alpha_{2}, \ldots, \alpha_{n}\right)^{T}, \beta=\left(\beta_{1}, \beta_{2}, \ldots, \beta_{n}\right)^{T}:[-\tau, T) \rightarrow \mathcal{R}^{n}$, such that $\alpha(t) \in K[f(x(t))], \beta(t) \in K[g(x(t))]$ for a.a $t \in[-\tau, T)$ and

$$
\dot{x}(t)=-D x(t)+A \alpha(t)+B \beta(t-\tau(t)) \text {, for a.a. } t \in[0, T) .
$$

Definition 3 (Forti et al., 2005). A vector $\eta \in \mathcal{R}^{n}$ is an equilibrium point of system (1) if it satisfies

$$
0 \in-D \eta+A K[f(\eta)]+B K[g(\eta)] .
$$

Equivalently, $\eta \in \mathcal{R}^{n}$ is an equilibrium point of system (1) if there exist $\zeta \in K[f(\eta)]$ and $\varsigma \in K[g(\eta)]$ such that

$$
-D \eta+A \zeta+B \varsigma=0
$$

Under Assumptions (A1) and (A2), we can see that the origin is one equilibrium point of system (1), i.e. $\eta=\zeta=\varsigma=0$. Next we give the definition of an initial value problem (IVP) associated to (1).

Definition 4 (IVP) (Forti et al., 2005). For any continuous function $\phi:[-\tau, 0] \rightarrow \mathcal{R}^{n}$ and any measurable selections $\chi(s) \in K[f(\phi(s))], \theta(s) \in K[g(\phi(s))]$ for a.a. $s \in[-\tau, 0]$ by an IVP associated to (1) with initial condition $(\phi, \chi, \theta)$, we mean the following problem: find a couple of functions $[x, \alpha, \beta]:[-\tau, T) \rightarrow \mathcal{R}^{n} \times \mathcal{R}^{n} \times \mathcal{R}^{n}$, such that $x$ is a solution of (1) on $[-\tau, T)$ for some $T>0, \alpha, \beta$ are the outputs associated to $x$, and

$$
\left\{\begin{aligned}
\dot{x}(t)= & -D x(t)+A \alpha(t)+B \beta(t-\tau(t)), \\
& \text { for a.a. } t \in[0, T) \\
\alpha(t) \in \quad & K[f(x(t))], \beta(t) \in K[g(x(t))], \\
& \text { for a.a. } t \in[0, T) \\
x(s)= & \phi(s), \forall s \in[-\tau, 0], \\
\alpha(s)= & \chi(s), \beta(s)=\theta(s), \text { for a.a. } s \in[-\tau, 0] .
\end{aligned}\right.
$$

Throughout this paper, we consider the controlled DNNs as follows:

$$
\dot{x}(t)=-D x(t)+A f(x(t))+B g(x(t-\tau(t)))+u(t)
$$

where $x(t)=\left(x_{1}(t), x_{2}(t), \ldots, x_{n}(t)\right)^{T} \in \mathcal{R}^{n}$ is the state vector. $u(t)=\left(u_{1}(t), u_{2}(t), \ldots, u_{n}(t)\right)^{T} \in \mathcal{R}^{n}$ is the control input vector, the other parameters are the same as in system (1). From now on we will consider the solution $[x, \alpha, \beta]$ of any IVP similarly defined as in (9).

Lemma 1 (Guo \& Huang, 2009). Suppose that the Assumptions (A1) and (A2) are satisfied, then there exist at least one solution for the IVP of system (1) defined on $[0,+\infty)$.

For further analysis we need the following definitions and lemmas. Let $\Lambda$ be an open subset of $C\left([-\tau, 0], \mathcal{R}^{n}\right)$ containing 0 .

Definition 5 (Moulay et al., 2008). The origin of system (1) is finite-time stable, if

(i) The origin of system (1) is stable;

(ii) The origin of system (1) is finite-time convergent, i.e. for any initial state $\phi(s) \in \Lambda$, there exists $0 \leq T(\phi)<+\infty$ such that every solution of system (1) satisfies $x(t, \phi)=0$ for all $t \geq T(\phi)$. $T_{0}(\phi)=\inf \{T(\phi) \geq 0: x(t, \phi)=0 \quad \forall t \geq T(\phi)\}$ is a functional called the settling time of the system (1).

Definition 6. The system (1) with the parameter ranges defined by (2) is finite-time robust stabilizable if there exists a feedback controller $u(t)$ such that the controlled system (10) is finite-time stable for all $D \in D_{I}, A \in A_{I}$ and $B \in B_{I}$. The system (1) without the parameter ranges is finite-time stabilizable if there exists a feedback controller $u(t)$ such that the controlled system (10) is finite-time stable.

Definition 7 (Forti et al., 2006). A function $V(x): \mathcal{R}^{n} \rightarrow \mathcal{R}$, which is locally Lipschitz near $x \in \mathcal{R}^{n}$, is said to be regular if the following holds. For all direction $v \in \mathcal{R}^{n}$, there exists the usual right-sided directional derivative

$$
V^{\prime}(x, v)=\lim _{h \rightarrow 0^{+}} \frac{V(x+h v)-V(x)}{h}
$$

and we have $V^{\prime}(x, v)=V^{0}(x, v)$, where

$$
V^{0}(x, v)=\limsup _{y \rightarrow x, h \rightarrow 0^{+}} \frac{V(y+h v)-V(y)}{h}
$$

is the generalized directional derivative of $V$ at $x$ in the direction $v$. The function $V$ is said to be regular in $\mathcal{R}^{n}$, if it is regular for any $x \in \mathcal{R}^{n}$.

Lemma 2 (Chain Rule, Forti et al. (2006)). If $V(x): \mathcal{R}^{n} \rightarrow \mathcal{R}$ is Clarke-regular (regular, positive definite and radially unbounded), and $x(t)$ is absolutely continuous on any compact subinterval of $[0,+\infty)$. Then, $x(t)$ and $V(x(t)):[0,+\infty) \rightarrow \mathcal{R}$ are differentiable for a.a. $t \in[0,+\infty)$ and

$$
\frac{\mathrm{d}}{\mathrm{d} t} V(x(t))=\breve{\gamma}^{T}(t) \dot{x}(t), \forall \breve{\gamma} \in \partial V(x(t)),
$$

where $\partial V(x)=c o\left\{\lim _{i \rightarrow \infty} \nabla V\left(x_{i}\right): x_{i} \rightarrow x, x_{i} \notin S \cup \Omega_{V}\right\}$ is the Clarke generalized gradient of $V$ at $x(t), c o(\cdot)$ denotes the convex hull, $S \subset \mathcal{R}^{n}$ is a set of measure zero and $\Omega_{V} \subset \mathcal{R}^{n}$ is a set of points at which function $V$ fails to be differentiable.

Lemma 3 (Haddad \& Nersesov, 2008). If there exist a continuous function $V:[0,+\infty) \times \Lambda \rightarrow \mathcal{R}_{+}$and two functions $v, r$ of class $\mathcal{K}$ for the controlled system (10) such that 
(i) $V(t, 0)=0, v(\|x\|) \leq V(t, x), t \in[0,+\infty)$,

(ii) $D^{+} V(t, x) \leq-r(V(t, x))$ with $\int_{0}^{\varepsilon} \frac{\mathrm{d} z}{r(z)}<+\infty$,

for all $\varepsilon>0, x \in \Lambda$. Then system (10) is finite-time stable with a settling time satisfying the inequality $T_{0}(\phi) \leq \int_{0}^{V(0, \phi)} \frac{\mathrm{d} z}{r(z)}$. In particular, if $r(V)=\lambda V^{\rho}$ where $\lambda>0, \rho \in(0,1)$, then the settling time satisfies the inequality

$$
T_{0}(\phi) \leq \int_{0}^{V(0, \phi)} \frac{\mathrm{d} z}{r(z)}=\frac{V^{1-\rho}(0, \phi)}{\lambda(1-\rho)} .
$$

Lemma 4 (Hardy et al., 1988). If $a_{1}, a_{2}, \ldots, a_{n}, \eta_{1}, \eta_{2}$ are real numbers and $0<\eta_{1}<\eta_{2}$, then the following inequality holds

$$
\left[\sum_{i=1}^{n}\left(\left|a_{i}\right|\right)^{\eta_{2}}\right]^{1 / \eta_{2}} \leq\left[\sum_{i=1}^{n}\left(\left|a_{i}\right|\right)^{\eta_{1}}\right]^{1 / \eta_{1}} .
$$

Lemma 5 (Berman \& Plemmons, 1979). Let $C \in Z$, where $C=\left(c_{i j}\right)$ denotes an $n \times n$ matrix and $Z$ denotes the class of square matrices with nonpositive off diagonal element. Each of the following conditions is equivalent to that $C$ is a nonsingular $M$-matrix.

1) All principal minors of $C$ are positive.

2) All diagonal elements of $C$ are positive and there exists a positive diagonal matrix $Q=\operatorname{diag}\left(q_{1}, q_{2}, \ldots, q_{n}\right)$ such that $C Q$ is strictly diagonally row dominant; i.e.,

$$
c_{i i} q_{i}>\sum_{j=1, j \neq i}^{n}\left|c_{i j}\right| q_{j}, \quad i=1,2, \ldots, n .
$$

3) All diagonal elements of $C$ are positive and there exists a positive diagonal matrix $Q=\operatorname{diag}\left(q_{1}, q_{2}, \ldots, q_{n}\right)$ such that $P C$ is strictly diagonally column dominant; i.e.,

$$
c_{j j} q_{j}>\sum_{i=1, i \neq j}^{n} q_{i}\left|c_{i j}\right|, \quad j=1,2, \ldots, n .
$$

\section{Main results}

In this section, our aim is to design a feedback controller so as to realize the finite-time robust stabilization of DNNs with discontinuous activations and parameter uncertainties. A delayed feedback control law is given as follows:

$$
\begin{aligned}
u_{i}(t)= & -k_{1 i} x_{i}(t)-k_{2 i} \operatorname{sgn}\left(x_{i}(t)\right) \sum_{j=1}^{n}\left|x_{j}\left(t-\tau_{j}(t)\right)\right| \\
& -k_{3 i} \operatorname{sgn}\left(x_{i}(t)\right), i=1,2, \ldots, n
\end{aligned}
$$

where $k_{1 i}>0, k_{2 i}>0, k_{3 i}>0$ are constants to be determined.

Under the controller (14), the controlled system (10) is transformed into:

$$
\begin{gathered}
\dot{x}(t)=-D x(t)+A \alpha(t)+B \beta(t-\tau(t))+\omega(t), \\
\text { for a.a. } t \in[0, T)
\end{gathered}
$$

where $\alpha(t) \in K[f(x(t))], \beta(t) \in K[g(x(t))], \omega(t)=$ $\left(\omega_{1}(t), \ldots, \omega_{n}(t)\right)^{T} \in K[u(t)]$, for a.a. $t \in[0, T)$. And $K[u(t)]=\left(K\left[u_{1}(t)\right], \ldots, K\left[u_{n}(t)\right]\right)^{T}, K\left[u_{i}(t)\right]=-k_{1 i} x_{i}(t)-$ $k_{2 i} K\left[\operatorname{sgn}\left(x_{i}(t)\right)\right] \sum_{j=1}^{n}\left|x_{j}\left(t-\tau_{j}(t)\right)\right|-k_{3 i} K\left[\operatorname{sgn}\left(x_{i}(t)\right)\right]$ with

$$
K\left[\operatorname{sgn}\left(x_{i}(t)\right)\right]= \begin{cases}1, & \text { if } x_{i}(t)>0, \\ {[-1,1],} & \text { if } x_{i}(t)=0, \\ -1, & \text { if } x_{i}(t)<0 .\end{cases}
$$

For presentation convenience, in the following, we denote $\hat{a}_{i j}=\max \left\{\left|\underline{a}_{i j}\right|,\left|\bar{a}_{i j}\right|\right\}, \hat{b}_{i j}=\max \left\{\left|\underline{b}_{i j}\right|,\left|\bar{b}_{i j}\right|\right\}, \hat{A}=\left(\hat{a}_{i j}\right)_{n \times n}$,

$P_{1}=\operatorname{diag}\left(\sum_{j=1}^{n} \hat{a}_{1 j} h_{j}, \sum_{j=1}^{n} \hat{a}_{2 j} h_{j}, \ldots, \sum_{j=1}^{n} \hat{a}_{n j} h_{j}\right)$,

$P_{2}=\operatorname{diag}\left(\sum_{j=1}^{n} \hat{b}_{1 j} l_{j}, \sum_{j=1}^{n} \hat{b}_{2 j} l_{j}, \ldots, \sum_{j=1}^{n} \hat{b}_{n j} l_{j}\right)$,

$P_{3}=\operatorname{diag}\left(\sum_{j=1}^{n}\left(\hat{a}_{1 j} r_{j}+\hat{b}_{1 j} s_{j}\right), \ldots, \sum_{j=1}^{n}\left(\hat{a}_{n j} r_{j}+\hat{b}_{n j} s_{j}\right)\right)$,

$Q_{1}=\operatorname{diag}\left(\sum_{j=1}^{n}\left|a_{1 j}\right| h_{j}, \sum_{j=1}^{n}\left|a_{2 j}\right| h_{j}, \ldots, \sum_{j=1}^{n}\left|a_{n j}\right| h_{j}\right)$,

$Q_{2}=\operatorname{diag}\left(\sum_{j=1}^{n}\left|b_{1 j}\right| l_{j}, \sum_{j=1}^{n}\left|b_{2 j}\right| l_{j}, \ldots, \sum_{j=1}^{n}\left|b_{n j}\right| l_{j}\right)$,

$Q_{3}=\operatorname{diag}\left(\sum_{j=1}^{n}\left(\left|a_{1 j}\right| r_{j}+\left|b_{1 j}\right| s_{j}\right), \ldots, \sum_{j=1}^{n}\left(\left|a_{n j}\right| r_{j}+\left|b_{n j}\right| s_{j}\right)\right)$,

$R=\operatorname{diag}\left(\sum_{j=1}^{n}\left|a_{1 j}\right| r_{j}, \sum_{j=1}^{n}\left|a_{2 j}\right| r_{j}, \ldots, \sum_{j=1}^{n}\left|a_{n j}\right| r_{j}\right)$,

$H=\operatorname{diag}\left(h_{1}, h_{2}, \ldots, h_{n}\right), K_{1}=\operatorname{diag}\left(k_{11}, k_{12}, \ldots, k_{1 n}\right)$,

$K_{2}=\operatorname{diag}\left(k_{21}, k_{22}, \ldots, k_{2 n}\right), K_{3}=\operatorname{diag}\left(k_{31}, k_{32}, \ldots, k_{3 n}\right)$.

Theorem 1. Under assumptions (A1) and (A2), if $F_{1}=2(\underline{D}+$ $\left.K_{1}\right)-P_{1}-\hat{A} H$ is a nonsingular $M$-matrix, $F_{2}=n K_{2}-P_{2}$ and $F_{3}=K_{3}-P_{3}$ are two positive diagonal matrices, then system (1) is finite-time robust stabilizable via the controller (14).

Proof. If $F_{1}$ is a nonsingular $M$-matrix, then from Lemma 5, there exists $\xi_{i}>0$, such that for all $i=1,2, \ldots, n$, we have

$$
2 \xi_{i}\left(\underline{d}_{i}+k_{1 i}\right)-\sum_{j=1}^{n}\left(\xi_{i} \hat{a}_{i j} h_{j}+\xi_{j} \hat{a}_{j i} h_{i}\right)>0 .
$$

Since $F_{2}$ and $F_{3}$ are two positive diagonal matrices, then we have

$$
\begin{aligned}
& \xi_{i}\left(n k_{2 i}-\sum_{j=1}^{n} \hat{b}_{i j} l_{j}\right)>0, \\
& \xi_{i}\left(k_{3 i}-\sum_{j=1}^{n}\left(\hat{a}_{i j} r_{j}+\left|\hat{b}_{i j}\right| s_{j}\right)\right)>0 .
\end{aligned}
$$

Consider the following Lyapunov function

$$
V(t)=\sum_{i=1}^{n} \xi_{i} x_{i}^{2}(t)
$$

By calculating the time derivative of $V(t)$ along the trajectory of system (15), we get from Lemma 2 that for a.a. $t \in[0,+\infty)$

$\dot{V}(t)=2 \sum_{i=1}^{n} \xi_{i} x_{i}(t) \dot{x}_{i}(t)$ 


$$
\begin{aligned}
= & 2 \sum_{i=1}^{n} \xi_{i} x_{i}(t)\left[-d_{i} x_{i}(t)+\sum_{j=1}^{n} a_{i j} \alpha_{j}(t)+\sum_{j=1}^{n} b_{i j} \beta_{j}\left(t-\tau_{j}(t)\right)\right. \\
& \left.-k_{1 i} x_{i}(t)-\sum_{j=1}^{n} k_{2 i} \omega_{i}(t)\left|x_{j}\left(t-\tau_{j}(t)\right)\right|-k_{3 i} \omega_{i}(t)\right] \\
= & -2 \sum_{i=1}^{n} \xi_{i}\left(d_{i}+k_{1 i}\right) x_{i}^{2}(t)+2 \sum_{i=1}^{n} \sum_{j=1}^{n} \xi_{i} a_{i j} x_{i}(t) \alpha_{j}(t) \\
& +2 \sum_{i=1}^{n} \sum_{j=1}^{n} \xi_{i} b_{i j} x_{i}(t) \beta_{j}\left(t-\tau_{j}(t)\right)-2 \sum_{i=1}^{n} \xi_{i} k_{3 i}\left|x_{i}(t)\right| \\
& -2 \sum_{i=1}^{n} \sum_{j=1}^{n} \xi_{i} k_{2 i}\left|x_{i}(t)\right|\left|x_{j}\left(t-\tau_{j}(t)\right)\right|
\end{aligned}
$$

where $\omega_{i}(t)=\operatorname{sgn}\left(x_{i}(t)\right)$, if $x_{i}(t) \neq 0$, while $\omega_{i}(t)$ can be arbitrarily chosen in $[-1,1]$, if $x_{i}(t)=0$.

Based on the assumptions (A1) and (A2), we can get

$$
\begin{aligned}
& 2 \sum_{i=1}^{n} \sum_{j=1}^{n} \xi_{i} a_{i j} x_{i}(t) \alpha_{j}(t) \\
\leq & 2 \sum_{i=1}^{n} \sum_{j=1}^{n} \xi_{i}\left|a_{i j}\right|\left|x_{i}(t)\right|\left|\alpha_{j}(t)\right| \\
\leq & 2 \sum_{i=1}^{n} \sum_{j=1}^{n} \xi_{i} \hat{a}_{i j}\left|x_{i}(t)\right|\left(h_{j}\left|x_{j}(t)\right|+r_{j}\right) \\
\leq & 2 \sum_{i=1}^{n} \sum_{j=1}^{n} \xi_{i} \hat{a}_{i j} h_{j}\left|x_{i}(t)\right|\left|x_{j}(t)\right|+2 \sum_{i=1}^{n} \sum_{j=1}^{n} \xi_{i} \hat{a}_{i j} r_{j}\left|x_{i}(t)\right| \\
\leq & \sum_{i=1}^{n} \sum_{j=1}^{n}\left(\xi_{i} \hat{a}_{i j} h_{j}+\xi_{j} \hat{a}_{j i} h_{i}\right) x_{i}^{2}(t)+2 \sum_{i=1}^{n} \sum_{j=1}^{n} \xi_{i} \hat{a}_{i j} r_{j}\left|x_{i}(t)\right|,
\end{aligned}
$$

and

$$
\begin{aligned}
& 2 \sum_{i=1}^{n} \sum_{j=1}^{n} \xi_{i} b_{i j} x_{i}(t) \beta_{j}\left(t-\tau_{j}(t)\right) \\
\leq & 2 \sum_{i=1}^{n} \sum_{j=1}^{n} \xi_{i} \hat{b}_{i j} l_{j}\left|x_{i}(t)\right|\left|x_{j}\left(t-\tau_{j}(t)\right)\right|+2 \sum_{i=1}^{n} \sum_{j=1}^{n} \xi_{i} \hat{b}_{i j} s_{j}\left|x_{i}(t)\right| .
\end{aligned}
$$

By combining (16)-(18) and (20)-(22), we derive

$$
\begin{aligned}
\dot{V}(t) \leq & -\sum_{i=1}^{n} x_{i}^{2}(t)\left[2 \xi_{i}\left(\underline{d}_{i}+k_{1 i}\right)-\sum_{j=1}^{n}\left(\xi_{i} \hat{a}_{i j} h_{j}+\xi_{j} \hat{a}_{j i} h_{i}\right)\right] \\
& -2 \sum_{i=1}^{n} \sum_{j=1}^{n} \xi_{i}\left(k_{2 i}-\hat{b}_{i j} l_{j}\right)\left|x_{i}(t) \| x_{j}\left(t-\tau_{j}(t)\right)\right| \\
& -2 \sum_{i=1}^{n} \xi_{i}\left|x_{i}(t)\right|\left[k_{3 i}-\sum_{j=1}^{n} \hat{a}_{i j} r_{j}-\sum_{j=1}^{n} \hat{b}_{i j} s_{j}\right] \\
\leq & -2 \sum_{i=1}^{n} \delta_{i} \xi_{i}\left|x_{i}(t)\right| \\
\leq & -2 \delta \sum_{i=1}^{n} \xi_{i}\left|x_{i}(t)\right|
\end{aligned}
$$

where $\delta=\min _{i}\left\{\delta_{i}\right\}, \delta_{i}=k_{3 i}-\sum_{j=1}^{n}\left(\hat{a}_{i j} r_{j}+\hat{b}_{i j} s_{j}\right)>0$.

Based on Lemma 4 , we obtain the following inequality

$$
-\sum_{i=1}^{n} \xi_{i}^{1 / 2}\left|x_{i}(t)\right| \leq-\left[\sum_{i=1}^{n} \xi_{i}\left|x_{i}(t)\right|^{2}\right]^{1 / 2}
$$

Then we have

$$
\dot{V}(t) \leq-2 \delta \xi^{1 / 2} V^{1 / 2}(t),
$$

where $\xi=\min _{i}\left\{\xi_{i}\right\}$. On the other hand, we have

$$
\int_{0}^{\varepsilon} \frac{\mathrm{d} z}{2 \delta \xi^{1 / 2} z^{1 / 2}}=\frac{\varepsilon^{1 / 2}}{\delta \xi^{1 / 2}}<+\infty,
$$

for all $\varepsilon>0$.

From Lemma 3, we can conclude that system (15) is finitetime stable. That is to say, system (1) is finite-time robust stabilizable via the controller (14). The proof is completed.

Corollary 1. Under assumptions (A1) and (A2), if there exist constants $k_{1 i}>0, k_{2 i}>0, k_{3 i}>0$ such that for all $i=1,2, \ldots, n$,

$$
\begin{aligned}
& 2\left(\underline{d}_{i}+k_{1 i}\right)-\sum_{j=1}^{n}\left(\hat{a}_{i j} h_{j}+\hat{a}_{j i} h_{i}\right)>0, \\
& n k_{2 i}-\sum_{j=1}^{n} \hat{b}_{i j} l_{j}>0, \\
& k_{3 i}-\sum_{j=1}^{n}\left(\hat{a}_{i j} r_{j}+\hat{b}_{i j} s_{j}\right)>0 .
\end{aligned}
$$

Then system (1) is finite-time robust stabilizable via the controller (14), and the settling time for stabilization satisfies $T_{0}(\phi) \leq\|\phi\| / \delta$, where $\delta=\min _{i}\left\{\delta_{i}\right\}, \delta_{i}=k_{3 i}-\sum_{j=1}^{n}\left(\hat{a}_{i j} r_{j}+\right.$ $\left.\hat{b}_{i j} s_{j}\right), i=1,2, \ldots, n$.

When $D=\underline{D}=\bar{D}, A=\underline{A}=\bar{A}, B=\underline{B}=\bar{B}$, i.e. the system (1) is without the parameter uncertainties, then we have the following results.

Theorem 2. Under assumptions (A1) and (A2), if $G_{1}=2(D+$ $\left.K_{1}\right)-Q_{1}-|A| H$ is a nonsingular $M$-matrix, $G_{2}=n K_{2}-Q_{2}$ and $G_{3}=K_{3}-Q_{3}$ are two positive diagonal matrices, then system (1) is finite-time stabilizable via the controller (14).

Corollary 2. Under assumptions (A1) and (A2), if there exist constants $k_{1 i}>0, k_{2 i}>0, k_{3 i}>0$ such that for all $i=1,2, \ldots, n$,

$$
\begin{aligned}
& 2\left(d_{i}+k_{1 i}\right)-\sum_{j=1}^{n}\left(\left|a_{i j}\right| h_{j}+\left|a_{j i}\right| h_{i}\right)>0, \\
& n k_{2 i}-\sum_{j=1}^{n}\left|b_{i j}\right| l_{j}>0 \\
& k_{3 i}-\sum_{j=1}^{n}\left(\left|a_{i j}\right| r_{j}+\left|b_{i j}\right| s_{j}\right)>0 .
\end{aligned}
$$

Then system (1) is finite-time stabilizable via the controller (14), and the settling time for stabilization satisfies $T_{0}(\phi) \leq$ 
$\|\phi\| / \delta$, where $\delta=\min _{i}\left\{\delta_{i}\right\}, \delta_{i}=k_{3 i}-\sum_{j=1}^{n}\left(\left|a_{i j}\right| r_{j}+\left|b_{i j}\right| s_{j}\right), i=$ $1,2, \ldots, n$.

Remark 1. In this paper, finite time stabilization of DNNs is realized. Our finite-time stability results are general compared with these infinite time (asymptotic or exponential) results in Huang et al. (2013); Phat \& Trinh (2010); Shen \& Wang (2009); Wang \& Liu (2005); Wang \& Shen (2014); Wang et al. (2015a); Wen et al. (2015); Wu \& Zeng (2012); Zhang \& Shen (2015).

Remark 2. System (1) with discontinuous right-hand side in this paper is general in view of the fact that it is with delays and parameter uncertainties. In Theorem 2 we also give the finite-time stabilization algebraic for neural networks without parameter uncertainties. In Liu et al. (2014a), finite-time stabilization of neural networks with discontinuous activations was studied and in Liu et al. (2014b), finite-time stabilization of Lipschitzain nonlinear systems with application to neural networks was studied. However, the systems in the two papers are without delays and parameter uncertainties. So our methods are general and can be used to study finite-time stability of other nonlinear delayed systems with or without parameter uncertainties.

When $D=\underline{D}=\bar{D}, A=\underline{A}=\bar{A}, B=\underline{B}=\bar{B}=0$, i.e. the system (1) is without the parameter uncertainties and delays and has the following form:

$$
\dot{x}(t)=-D x(t)+A f(x(t)),
$$

where $x(t)=\left(x_{1}(t), x_{2}(t), \ldots, x_{n}(t)\right)^{T} \in \mathcal{R}^{n}$ is the state vector, the other parameters are the same as in system (1). Then we have the following results.

Theorem 3. Under assumptions (A1) and (A2), if $G_{1}=2(D+$ $\left.K_{1}\right)-Q_{1}-|A| H$ is a nonsingular $M$-matrix and $G_{3}=K_{3}-$ $R$ is a positive diagonal matrix, then system (32) is finite-time stabilizable via the controller

$$
u_{i}(t)=-k_{1 i} x_{i}(t)-k_{3 i} \operatorname{sgn}\left(x_{i}(t)\right), i=1,2, \ldots, n .
$$

Corollary 3. Under assumptions (A1) and (A2), if there exist constants $k_{1 i}>0, k_{3 i}>0$ such that for all $i=1,2, \ldots, n$,

$$
\begin{aligned}
& 2\left(d_{i}+k_{1 i}\right)-\sum_{j=1}^{n}\left(\left|a_{i j}\right| h_{j}+\left|a_{j i}\right| h_{i}\right)>0, \\
& k_{3 i}-\sum_{j=1}^{n}\left|a_{i j}\right| r_{j}>0 .
\end{aligned}
$$

Then system (32) is finite-time stabilizable via the controller (33), and the settling time for stabilization satisfies $T_{0}(x(0)) \leq$ $\|x(0)\| / \delta$, where $x(0)$ is the initial condition of system (32), $\delta=$ $\min _{i}\left\{\delta_{i}\right\}, \delta_{i}=k_{3 i}-\sum_{j=1}^{n}\left|a_{i j}\right| r_{j}, i=1,2, \ldots, n$.

Remark 3. The main results in Liu et al. (2014a) gives the verifiable criteria for finite-time stabilization of neural networks with discontinuous activations as follows: there exist positive definite matrix $P \in \mathcal{R}^{n \times n}$ and constants $k_{1}>0, k_{3}>0$ such that

$$
k_{1}-n \max _{1 \leq i \leq n}\left\{h_{i}\right\} \tilde{a} / \lambda_{\min }(P)>0
$$

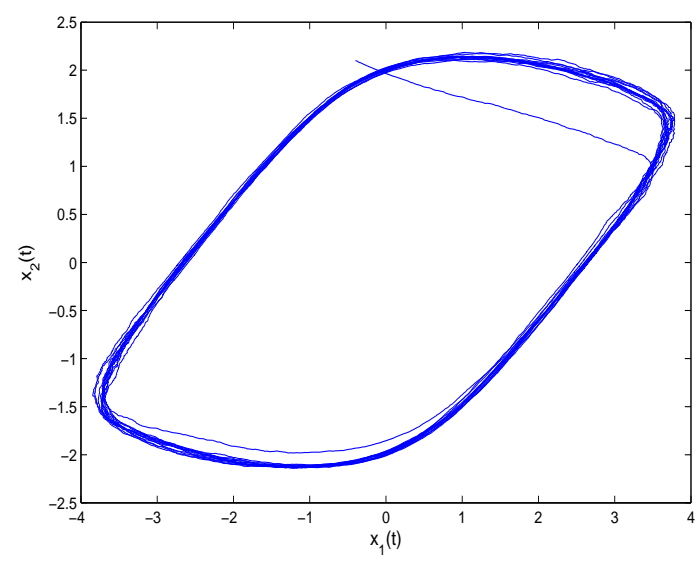

Figure 1: Phase plot of system (36) with the initial condition $x_{1}(s)=$ $-0.4, x_{2}(s)=2.1, \forall s \in[-1,0)$.

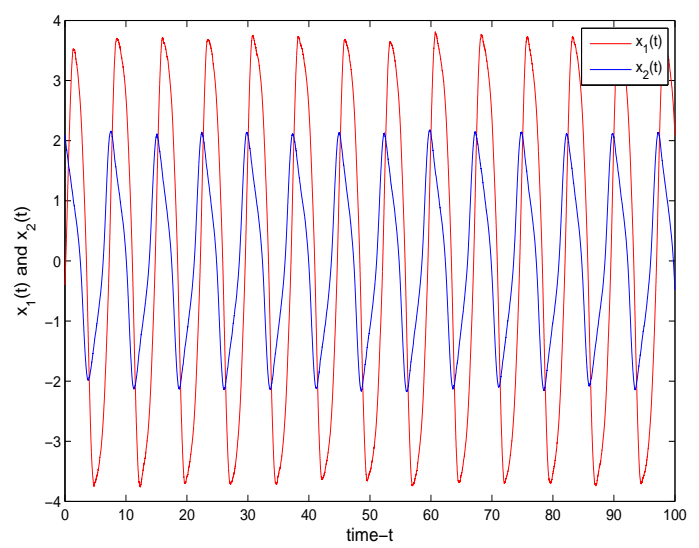

Figure 2: State trajectories of variables $x_{1}(t)$ and $x_{2}(t)$ of system (36) with the initial condition $x_{1}(s)=-0.4, x_{2}(s)=2.1, \forall s \in[-1,0)$.

$$
k_{3}-n \max _{1 \leq i \leq n}\left\{r_{i}\right\} / \lambda_{\min }(P)>0
$$

where $\tilde{a}=\max _{1 \leq i, j \leq n}\left\{\left|\tilde{a}_{i j}\right|\right\}, P A=\left(\tilde{a}_{i j}\right)_{n \times n}$. The controller in Liu et al. (2014a) is $u_{i}(t)=-k_{1} x_{i}(t)-k_{3} \operatorname{sgn}\left(x_{i}(t)\right), i=1,2, \ldots, n$.

Then for all $i=1,2, \ldots, n$, we have

$$
\begin{aligned}
& n \max _{1 \leq i \leq n}\left\{h_{i}\right\} \tilde{a} / \lambda_{\min }(P)>n \max _{1 \leq i, j \leq n}\left\{\left|a_{i j}\right|\right\} \max _{1 \leq i \leq n}\left\{h_{i}\right\} \\
> & \frac{1}{2} \sum_{j=1}^{n}\left(\left|a_{i j}\right| h_{j}+\left|a_{j i}\right| h_{i}\right)>\frac{1}{2} \sum_{j=1}^{n}\left(\left|a_{i j}\right| h_{j}+\left|a_{j i}\right| h_{i}\right)-d_{i}, \\
& n \max _{1 \leq i \leq n}\left\{r_{i}\right\} / \lambda_{\min }(P)>n \max _{1 \leq i, j \leq n}\left\{\left|a_{i j}\right|\right\} \max _{1 \leq i \leq n}\left\{r_{i}\right\}>\sum_{j=1}^{n}\left|a_{i j}\right| r_{j} .
\end{aligned}
$$

It is obviously that the range of the controller parameters in this paper are wider than that in Liu et al. (2014a), which means that the criteria (34) and (35) are less conservative than (34') and (35'). 


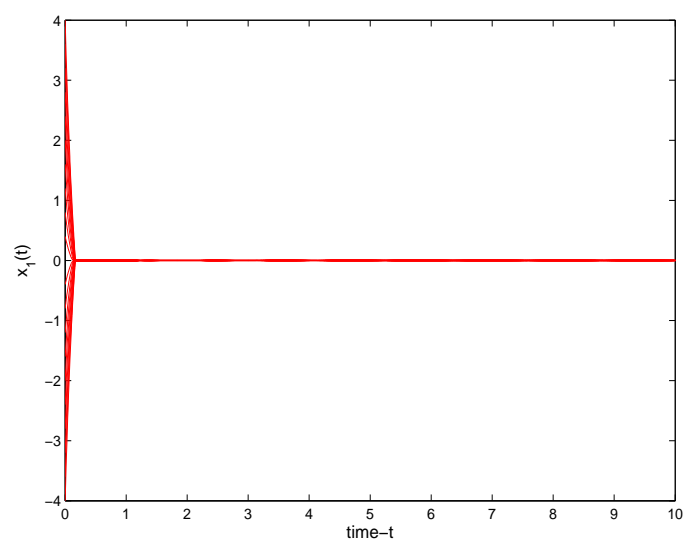

Figure 3: Choose 20 random initial conditions and time-domain behaviors of variable $x_{1}(t)$ of the controlled system (38) under the controller (37).

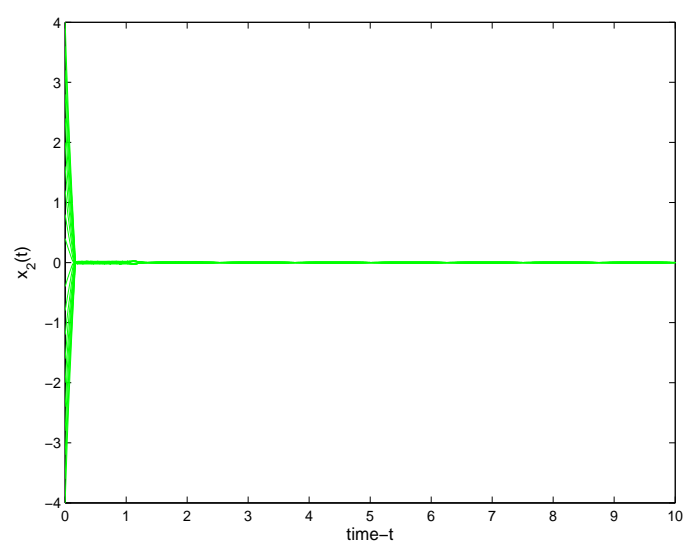

Figure 4: Choose 20 random initial conditions and time-domain behaviors of variable $x_{2}(t)$ of the controlled system (38) under the controller (37).

\section{Numerical simulations}

In this section, two examples are provided to verify the effectiveness of the results obtained in the previous section.

Example 1. Consider the two-dimensional uncertain DNNs

$$
\dot{x}(t)=-D x(t)+A f(x(t))+B g(x(t-\tau(t))),
$$

where $x(t)=\left(x_{1}(t), x_{2}(t)\right)^{T}, D \in D_{I}=[\underline{D}, \bar{D}], A \in A_{I}=$ $[\underline{A}, \bar{A}], B \in B_{I}=[\underline{B}, \bar{B}], \tau_{1}(t)=\tau_{2}(t)=e^{t} /\left(1+e^{t}\right)$,

$$
\begin{aligned}
& \underline{D}=\left[\begin{array}{cc}
0.3 & 0 \\
0 & 0.2
\end{array}\right], \bar{D}=\left[\begin{array}{cc}
1.1 & 0 \\
0 & 1.2
\end{array}\right], \\
& \underline{A}=\left[\begin{array}{cc}
1 & 1.2 \\
-0.1 & 2
\end{array}\right], \bar{A}=\left[\begin{array}{cc}
3 & 3.2 \\
0.1 & 3
\end{array}\right], \\
& \underline{B}=\left[\begin{array}{cc}
-2 & -0.2 \\
-0.1 & -3
\end{array}\right], \bar{B}=\left[\begin{array}{cc}
-1 & -0.1 \\
0.1 & -1
\end{array}\right] . \\
& f(x)= \begin{cases}\tanh (x)+0.01, & x>0, \\
\tanh (x)-0.01, & x<0 .\end{cases}
\end{aligned}
$$

$$
g(x)= \begin{cases}\tanh (x)+0.03 x+0.01, & x>0 \\ \tanh (x)+0.03 x-0.01, & x<0\end{cases}
$$

Then from the assumption (A2) that $h_{j}=1, l_{j}=1.03, r_{j}=s_{j}=$ 0.02 for $j=1,2$. $H=\operatorname{diag}(1,1)$. Under the initial condition $x_{1}(s)=-0.4, x_{2}(s)=2.1, \forall s \in[-1,0)$, the phase plot of system (36) is shown in Fig. 1 and the state trajectories of variables $x_{1}(t)$ and $x_{2}(t)$ of system (36) are shown in Fig.2.

Choose the control gain matrices as $K_{1}=\operatorname{diag}(5,3), K_{2}=$ $\operatorname{diag}(1.5,2), K_{3}=\operatorname{diag}(0.5,0.5)$, then the controller $u(t)=$ $\left(u_{1}(t), u_{2}(t)\right)^{T}$ is as follows

$$
\left\{\begin{aligned}
u_{1}(t)= & -5 x_{1}(t)-1.5 \operatorname{sgn}\left(x_{1}(t)\right) \sum_{j=1}^{2}\left|x_{j}\left(t-\tau_{j}(t)\right)\right| \\
& -0.5 \operatorname{sgn}\left(x_{1}(t)\right), \\
u_{2}(t)= & -3 x_{2}(t)-2 \operatorname{sgn}\left(x_{2}(t)\right) \sum_{j=1}^{2}\left|x_{j}\left(t-\tau_{j}(t)\right)\right| \\
& -0.5 \operatorname{sgn}\left(x_{2}(t)\right) .
\end{aligned}\right.
$$

The controlled system is

$$
\dot{x}(t)=-D x(t)+A f(x(t))+B g(x(t-\tau(t)))+u(t),
$$

where $x(t)=\left(x_{1}(t), x_{2}(t)\right)^{T}$, the parameters are the same as in system (36) and the controller $u(t)$ is defined by (37).

Then we have

$$
\begin{aligned}
& F_{1}=2\left(\underline{D}+K_{1}\right)-P_{1}-\hat{A} H=\left[\begin{array}{cc}
1.4 & -3.2 \\
-0.1 & 0.3
\end{array}\right], \\
& F_{2}=2 K_{2}-P_{2}=\left[\begin{array}{cc}
0.734 & 0 \\
0 & 0.807
\end{array}\right], \\
& F_{3}=K_{3}-P_{3}=\left[\begin{array}{cc}
0.332 & 0 \\
0 & 0.376
\end{array}\right] .
\end{aligned}
$$

Obviously, $F_{1}$ is a nonsingular $M$-matrix, $F_{2}$ and $F_{3}$ are two positive diagonal matrices, then system (36) can be finite-time robust stabilized via the controller (37) according to Theorem 1. Setting $\xi_{i}=1(i=1,2, \ldots, n)$ in (19), then the settling time for robust stabilization satisfies $T_{0}(\phi) \leq \frac{\|\phi\|}{\delta}=6.4390$ according to Corollary 1. Under the controller (37) and 20 random initial conditions, the state trajectories of variables $x_{1}(t)$ and $x_{2}(t)$ of the controlled system (38) are shown in Fig.3 and Fig.4, respectively.

Remark 4. As is pointed out in Forti \& Nistri (2003); Forti et al. (2005), neural networks with discontinuous neuron activations is an ideal model for the case where the gain of the neuron amplifiers is very high, and is frequently encountered in some applications. The general discontinuous activations can provide the richness of flexibility and opportunities in practical applications for the system.

Example 2. Consider the two-dimensional DNNs

$$
\dot{x}(t)=-D x(t)+A f(x(t))+B g(x(t-\tau(t))),
$$

where $x(t)=\left(x_{1}(t), x_{2}(t)\right)^{T}, D=\operatorname{diag}(1,1), \tau_{1}(t)=\tau_{2}(t)=$ $e^{t} /\left(1+e^{t}\right)$

$$
A=\left[\begin{array}{cc}
3 & 5 \\
0.1 & 2
\end{array}\right], B=\left[\begin{array}{cc}
-2.5 & 0.2 \\
0.1 & -1.5
\end{array}\right] .
$$




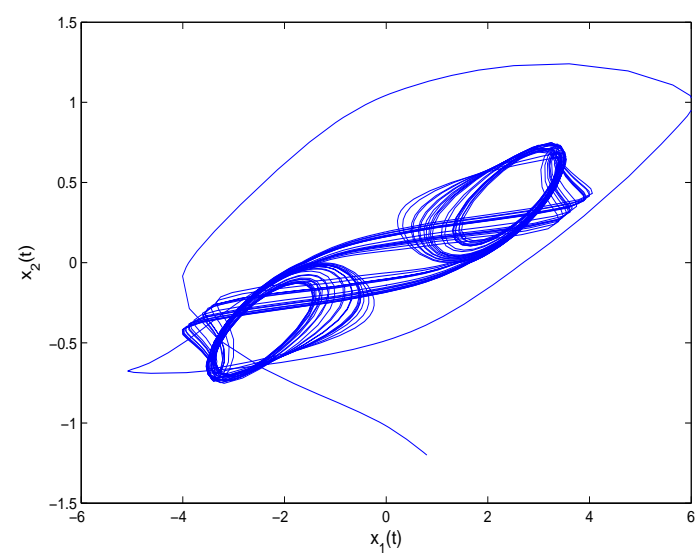

Figure 5: Phase plot of system (39) with the initial condition $x_{1}(s)=$ $0.8, x_{2}(s)=-1.2, \forall s \in[-1,0)$.

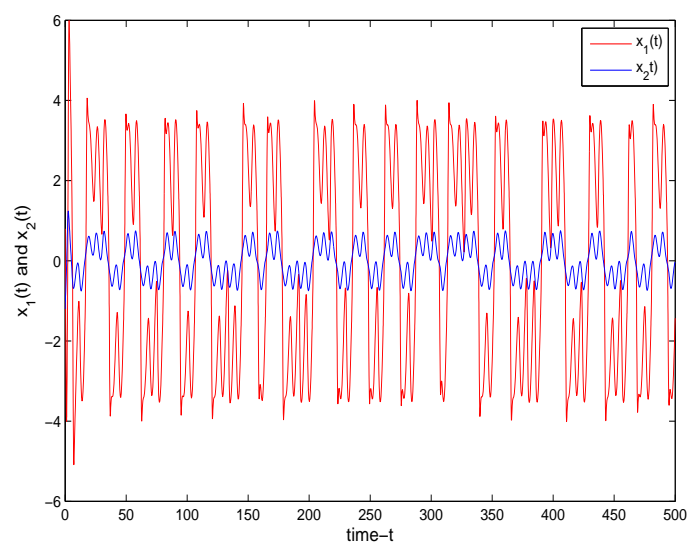

Figure 6: State trajectories of variables $x_{1}(t)$ and $x_{2}(t)$ of system (39).

$$
\begin{aligned}
& f(x)= \begin{cases}\tanh (x)+0.03, & x>0, \\
\tanh (x)-0.03, & x<0 .\end{cases} \\
& g(x)= \begin{cases}\tanh (x)+0.02 x+0.028, & x>0, \\
\tanh (x)+0.02 x-0.028, & x<0 .\end{cases}
\end{aligned}
$$

Then from the assumption (A2) that $h_{j}=1, l_{j}=1.02, r_{j}=$ $0.06, s_{j}=0.056$ for $j=1,2 . H=\operatorname{diag}(1,1),|A|=A$. Under the the initial condition $x_{1}(s)=0.8, x_{2}(s)=-1.2, \forall s \in[-1,0)$, the phase plot of system (39) is shown in Fig.5 and the state trajectories of variables $x_{1}(t)$ and $x_{2}(t)$ of system (39) are shown in Fig.6.

Choose the control gain matrices as $K_{1}=\operatorname{diag}(5,1.5), K_{2}=$ $\operatorname{diag}(1.5,1), K_{3}=\operatorname{diag}(1,0.5)$, then the controller $u(t)=$ $\left(u_{1}(t), u_{2}(t)\right)^{T}$ is designed as

$$
\left\{\begin{aligned}
u_{1}(t)= & -5 x_{1}(t)-1.5 \operatorname{sgn}\left(x_{1}(t)\right) \sum_{j=1}^{2}\left|x_{j}\left(t-\tau_{j}(t)\right)\right| \\
& -\operatorname{sgn}\left(x_{1}(t)\right), \\
u_{2}(t)= & -1.5 x_{2}(t)-\operatorname{sgn}\left(x_{2}(t)\right) \sum_{j=1}^{2}\left|x_{j}\left(t-\tau_{j}(t)\right)\right| \\
& -0.5 \operatorname{sgn}\left(x_{2}(t)\right) .
\end{aligned}\right.
$$

The controlled system is

$$
\dot{x}(t)=-D x(t)+A f(x(t))+B g(x(t-\tau(t)))+u(t),
$$

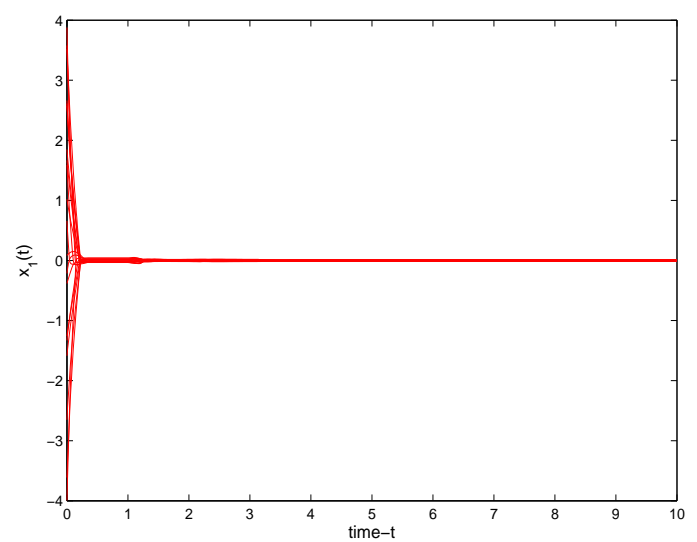

Figure 7: Choose 20 random initial conditions and time-domain behaviors of variable $x_{1}(t)$ of the controlled system (41) under the controller (40).

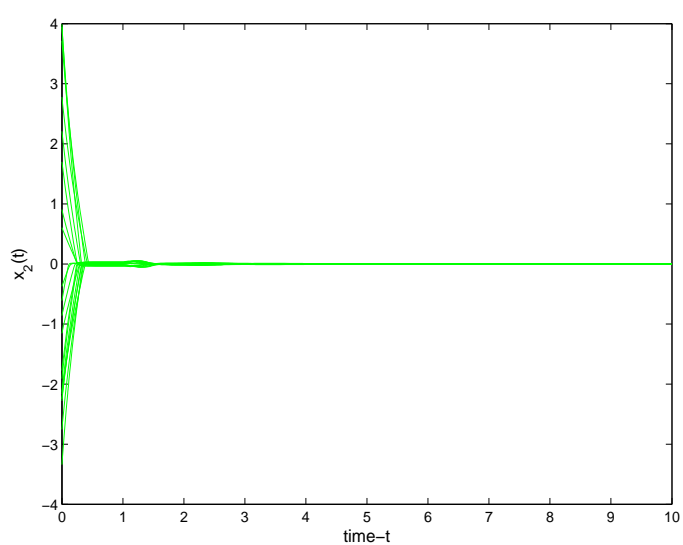

Figure 8: Choose 20 random initial conditions and time-domain behaviors of variable $x_{2}(t)$ of the controlled system (41) under the controller (40).

where $x(t)=\left(x_{1}(t), x_{2}(t)\right)^{T}$, the parameters are the same as in system (39) and the controller $u(t)$ is defined by (40).

Then we have

$$
\begin{aligned}
& G_{1}=2\left(D+K_{1}\right)-Q_{1}-|A| H=\left[\begin{array}{cc}
1 & -5 \\
-0.1 & 0.9
\end{array}\right], \\
& G_{2}=2 K_{2}-Q_{2}=\left[\begin{array}{cc}
0.246 & 0 \\
0 & 0.368
\end{array}\right], \\
& G_{3}=K_{3}-Q_{3}=\left[\begin{array}{cc}
0.3688 & 0 \\
0 & 0.2844
\end{array}\right] .
\end{aligned}
$$

Obviously, $G_{1}$ is a nonsingular $M$-matrix, $G_{2}$ and $G_{3}$ are two positive diagonal matrices, then system (39) can be finite-time stabilized via the controller (40) according to Theorem 2 . Then the settling time for stabilization satisfies $T_{0}(\phi) \leq \frac{\|\phi\|}{\delta}=$ 5.0711 according Corollary 2. Under the controller (40) and choose 20 random initial conditions, the state trajectories of variables $x_{1}(t)$ and $x_{2}(t)$ of the controlled system (41) are shown in Fig.7 and Fig.8, respectively. 


\section{Conclusions}

Finite-time robust stabilization for a class of DNNs with discontinuous activations and parameter uncertainties has been investigated in this paper. By designing a delayed controller, the finite-time robust stabilization algebraic criteria based on $M$ matrix have been established and the upper bound of the settling time functional has been estimated. Moreover, the finite-time stabilization criteria for DNNs without parameter uncertainties also have been given. The delayed control approach can be used to study the finite-time stability problem of other delayed systems with or without parameter uncertainties.

In this paper, the finite-time stability ensures the solution of the system be zero in a finite time. The time called the settling time functional depends on the initial condition heavily, which may brings some restrictions in practice. When the settling time functional is bounded, the problem refers to the fixed-time stability problem. In the future, we will study the fixed-time stability of DNNs with some other control approaches.

\section{Acknowledgments}

This work is supported by the Key Program of National Natural Science Foundation of China (Grant no. 61134012), the National Science Foundation of China (Grant nos. 11271146 and 61374150), and the Prior Development Field for the Doctoral Program of Higher Education of China (Grant no. 20130142130012).

\section{References}

Arik, S. (2014). An improved robust stability result for uncertain neural networks with multiple time delays, Neural Networks, 54, 1-10.

Berman, A., \& Plemmons, R. J. (1979). Nonnegative Matrices in the Mathematical Science, Academic Press, New York.

Bhat, S. P., \& Bernstein, D. S. (2000). Finite-time stability of continuous autonomous systems, SIAM Journal on Control and Optimization, 38, 751766.

Cao, J., \& Wang, J. (2005). Global exponential stability and periodicity of recurrent neural networks with time delays, IEEE Transactions on Circuits and systems I: Regular Papers, 52, 920-931.

Cohen, M. A., \& Grossberg, S. (1983). Absolute stability of global pattern formation and parallel memory storage by competitive neural networks, IEEE Transactions on Systems, Man and Cybernetics, 13, 815-826.

Efimov, D., Polyakov, A., Fridman, E., Perruquetti, E., \& Richard, J. P. (2014). Comments on finite-time stability of time-delay systems, Automatica, 50 1944-1947.

Faydasicok, O., \& Arik, S. (2012). Robust stability analysis of a class of neural networks with discrete time delays, Neural Networks, 29-30, 52-59.

Feng, W., Yang, S. X., \& Wu, H. (2015). Further results on robust stability of bidirectional associative memory neural networks with norm-bounded uncertainties, Neurocomputing, 148, 535-543.

Filippov, A. F. (1988). Differential Equations with Discontinuous Righthand Sides, Dordrecht: Kluwer.

Forti, M., \& Tesi, A. (1995). New conditions for global stability of neural networks with application to linear and quadratic programming problems, IEEE Transactions on Circuits and Systems I: Fundamental Theory and Applications, 42, 354-366.

Forti, M., \& Nistri, P. (2003). Global convergence of neural networks with discontinuous neuron activations, IEEE Transactions on Circuits and Systems I: Fundamental Theory and Applications, 50, 1421-1435.

Forti, M., Nistri, P., \& Papini, D. (2005). Global exponential stability and global convergence in finite time of delayed neural networks with infinite gain, IEEE Transactions on Neural Networks and Learning Systems, 16, 1449. 1463.
Forti, M., Grazzini, M., Nistri, P., \& Pancioni, L. (2006). Generalized Lyapunov approach for convergence of neural networks with discontinuous or nonLipschitz activations, Physica D: Nonlinear Phenomena, 214, 88-99.

Guo, Z., Wang, J., \& Yan, Z. (2014). A systematic method for analyzing robust stability of interval neural networks with time-delays based on stability criteria, Neural Networks, 54, 112-122.

Guo, Z., \& Huang, L. (2009). LMI conditions for global robust stability of delayed neural networks with discontinuous neuron activations, Applied Mathematics and Computation, 215, 889-900.

Haddad, W. M., Nersesov, S. G., \& Du, L. (2008) Finite-time stability for time-varying nonlinear dynamical systems, In American Control Conference, 4135-4139.

Hardy, G., Littlewood, J., \& Polya, G. (1988). Inequalities, Cambridge University Press: Cambridge.

Hong, Y., \& Jiang, Z. P. (2006). Finite-time stabilization of nonlinear systems with parametric and dynamic uncertainties, IEEE Transactions on Automatic Control, 51, 1950-1956.

Hu, C., Yu, J., \& Jiang, H. (2014). Finite-time synchronization of delayed neural networks with Cohen-Grossberg type based on delayed feedback control, Neurocomputing, 143, 90-96.

Huang, H., Huang, T., Chen, X., \& Qian, C. (2013). Exponential stabilization of delayed recurrent neural networks: A state estimation based approach, Nueral Networks, 48, 153-157.

Huang, J., Li, C., Huang, T., \& He, X. (2014). Finite-time lag synchronization of delayed neural networks, Neurocomputing, 139, 145-149.

Huang, T., Li, C., Duan, S. \& Starzyk, J. A. (2012). Robust exponential stability of uncertain delayed neural networks with stochastic perturbation and impulse effects, IEEE Transactions on Neural Networks and Learning Systems, 23, 866-875.

Karafyllis, I. (2006). Finite-time global stabilization by means of time-varying distributed delay feedback, SIAM Journal on Control and Optimization, 45, 320-342.

Liu, Y., Wang, Z., \& Liu, X. (2006). Global exponential stability of generalized recurrent neural networks with discrete and distributed delays, Neural Networks, 19, 667-675.

Liu, X., Park, J. H., Jiang, N., \& Cao, J. (2014a). Nonsmooth finite-time stabilization of neural networks with discontinuous activations, Nueral Networks, 52, 25-32.

Liu, X., Ho, D. W. C., Yu, W., \& Cao, J. (2014b). A new switching design to finite-time stabilization of nonlinear systems with applications to neural networks, Nueral Networks, 57, 94-102.

Moulay, E., Dambrine, M., Yeganefar, N., \& Perruquetti, W. (2008). Finitetime stability and stabilization of time-delay systems, Systems and Control Letters, 57, 561-566.

Phat, V. N., \& Trinh, H. (2010). Exponential stabilization of neural networks with various activation functions and mixed time-varying delays, IEEE Transactions on Neural Networks, 21, 1180-1184.

Shen, Y., \& Wang, J. (2009). Almost sure exponential stability of recurrent neural networks with Markovian switching, IEEE Transactions on Neural Networks, 20, 840-855.

Shen, Y., \& Wang, J. (2012). Robustness analysis of global exponential stability of recurrent neural networks in the presence of time delays and random disturbances, IEEE Transactions on Neural Networks and Learning Systems, 23, 87-96.

Sun, L., Feng, G., \& Wang, Y. (2014). Finite-time stabilization and H $\infty$ control for a class of nonlinear Hamiltonian descriptor systems with application to affine nonlinear descriptor systems, Automatica, 50, 2090-2097.

Wang, Z., Liu, Y., \& Liu, X. (2005). On global asymptotic stability of neural networks with discrete and distributed delays, Physics Letters A, 345, 299308.

Wang L., \& Xiao, F. (2010). Finite-time consensus problems for networks of dynamic agents, IEEE Transactions on Automatic Control, 55, 950-955.

Wang L., \& Shen, Y. (2014). New results on passivity analysis of memristorbased neural networks with time-varying delays, Neurocomputing, 144, 208 214.

Wang L., Shen, Y., Yin, Q., \& Zhang, G. (2015). Adaptive synchronization of memristor-based neural networks with time-varying delays, IEEE Transactions on Neural Networks and Learning Systems, 26, 2033-2042.

Wang, L., Shen, Y., \& Ding, Z. (2015). Finite time stabilization of delayed neural networks, Nueral Networks, 70, 74-80. 
Wang L., \& Shen, Y. (2015). Finite-time stabilizability and instabilizability of delayed memristive neural networks with nonlinear discontinuous controller, IEEE Transactions on Neural Networks and Learning Systems, 26, 2914-2924.

Wang, X., Li, C., \& Huang, T. (2014). Delay-dependent robust stability and stabilization of uncertain memristive delay neural networks, Neurocomputing, 140, 155-161.

Wen, S., Huang, T., Zeng, Z., Chen, Y., \& Li, P. (2015). Circuit design and exponential stabilization of memristive neural networks, Neural Networks, 63, 48-56.

Wu, A., \& Zeng, Z. (2012). Exponential stabilization of memristive neural networks with time delays, IEEE Transactions on Neural Networks and Learning Systems, 23, 1919-1929.

Wu, X., Wang, Y., Huang, L., \& Zuo, Y. (2010). Robust exponential stability criterion for uncertain neural networks with discontinuous activation functions and time-varying delays, Neurocomputing, 73, 1265-1271.

Xiao, J., \& Zeng, Z. (2014). Global robust stability of uncertain delayed neural networks with discontinuous neuron activation, Neural Computing and Applications, 24, 1191-1198.

Yang, R., \& Wang, Y. (2012). Finite-time stability and stabilization of a class of nonlinear time-delay systems, SIAM Journal on Control and Optimization, 50, 3113-3131.

Yang, R., Gao, H., \& Shi, P. (2009). Novel robust stability criteria for stochastic Hopfield neural networks with time delays, IEEE Transactions on Systems, Man, and Cybernetics, Part B: Cybernetics, 39, 467-474.

Yang, X. (2014). Can neural networks with arbitrary delays be finite-timely synchronized, Neurocomputing, 143, 275-281.

Zeng, Z., \& Zheng, W. X. (2012). Multistability of neural networks with timevarying delays and concave-convex characteristics, IEEE Transactions on Neural Networks and Learning Systems, 23, 293-305.

Zhang, G., \& Shen, Y. (2015). Exponential stabilization of memristor-based chaotic neural Networks with time-Varying delays via intermittent control, IEEE Transactions on Neural Networks and Learning Systems, 26, 14311441.

Zhang, H., Wang, Z., \& Liu, D. (2008). Robust stability analysis for interval Cohen-Grossberg neural networks with unknown time-varying delays, IEEE Transactions on Neural Networks, 19, 1942-1955.

Zuo, Y., Wang, Y., Huang, L., Wang, Z., Liu, X., \& Wu, X. (2009). Robust stability criterion for delayed neural networks with discontinuous activation functions, Neural Processing Letters, 29, 29-44. 